\title{
Consensus of Multiagent Systems with Packet Losses and Communication Delays Using a Novel Control Protocol
}

\author{
Zheping Yan, Di Wu, Wei Zhang, and Yibo Liu \\ College of Automation, Harbin Engineering University, Heilongjiang 150001, China \\ Correspondence should be addressed to Di Wu; roaddywu@gmail.com
}

Received 16 January 2014; Revised 23 February 2014; Accepted 24 February 2014; Published 7 April 2014

Academic Editor: Peng Shi

Copyright (C) 2014 Zheping Yan et al. This is an open access article distributed under the Creative Commons Attribution License, which permits unrestricted use, distribution, and reproduction in any medium, provided the original work is properly cited.

\begin{abstract}
This paper studies the consensus problem of multiagent system with packet losses and communication delays under directed communication channels. Different from previous research results, a novel control protocol is proposed depending only on periodic sampling and transmitting data in order to be convenient for practical implementation. Due to the randomicity of transmission delays and packet losses, each agent updates its input value asynchronously at discrete time instants with synchronized time stamped information and evolves in continuous time. Consensus conditions for multiagent system consists of three typical dynamics including single integrator, double integrator, and high-order integrator that are all discussed in this paper. It is proved that, for single integrator agents and double integrator systems with only communication delays, consensusability can be ensured through stochastic matrix theory if the designed communication topology contains a directed spanning tree. While, for double integrator agents and high-order integrator agents with packet losses and communication delays, the interval system theory is introduced to prove the consensus of multiagent system under the condition that the designed communication topology is a directed spanning tree. Finally, simulations are carried out to validate the effectiveness of the proposed solutions.
\end{abstract}

\section{Introduction}

Consensus of multiagent system has attracted increasing focuses of researchers from different areas including multiple robotics system, large-scale oceanographic survey, and wireless sensor networks [1-4]. Among all the problems studied aiming at achieving consensus through local interaction, packet losses and communication delays that usually result from unreliable communication links are significant factors which influence the consensusability of multiagent system [5-25]. Considering those problems, three typical agent dynamics, single integrator $[5,9,16,23]$, double integrator $[6,8,10,12,14,17,18]$, and high-order integrator $[11,15]$, are most widely discussed because they can represent a majority of autonomous systems.

Even though plenty of research results have been carried out about consensus problem of multiagent systems with communication delays, most of the controllers designed cannot be easily practiced. Take two typical controllers; for example, in Gao and Wang [10], the structure of controller implies that each agent has to memorize system states all the time between two sampling instants because of the randomicity of communication delay. In Lin and Jia [12], to deploy the control protocol, each agent has to broadcast the information all the time and only one transmitted data is useful to calculate the control input. In both cases, system resources are wasted to some extent, especially for embedded systems whose resources are ordinarily limited. So in this paper, in order to be convenient for practical implementation and economical for limited system resource, a novel controller structure is proposed depending only on periodic sampling and transmitting data which is the main contribution of this paper. Compared with previously proposed solutions, this protocol greatly relieves the computational burden of each agent. In addition, with this control protocol, it is worth being noticed that since the communication delays are random, each agent updates its input asynchronously at discrete time instants based on received data. But the information transmitted is with synchronized time stamp and the whole system evolves in continuous time.

With the proposed control protocol, interaction topology is time varying due to communication delays and packet 
losses. Also, many research results about consensus of multiagent systems with dynamically changing topology have been obtained based on stochastic matrix theory and Lyapunov theory. However, Lyapunov theory usually needs the topology to be undirected or balanced $[7,13,14]$. When random transmission delays and packet losses are concerned, undirected or balanced assumption is unreasonable. With the novel control protocol proposed in this paper, it will be shown that, for single integrator agent and second order agent without packet losses, similar results can be obtained as in $[8,9]$ according to stochastic matrix theory. That is, the consensus can be reached as long as the designed communication topology contains a directed spanning tree. However, when it comes to second order and high-order agent with packet losses and communication delays, stochastic theory is no longer easily applicable since the nonnegativity of the system matrix is not always guaranteed. Actually, to the best of the authors' knowledge, few results have been obtained to handle this problem. Based on the theory of interval matrix, it will be proved that the consensus of the system can be reached as long as the designed communication topology is a directed spanning tree which is another contribution of this paper. Of course, in this situation, the states of all agents will converge to the root node and average consensus cannot be obtained. However, this assumption can also simplify the system architecture indicating that one agent can only receive message from its superior and send information to its inferiors; this kind of hierarchy structure is actually more efficient and convenient in real-world application. Since the protocol proposed in this paper can be easily implemented for system with limited resources and unreliable communication links, it would have a wide application prospect in multiple autonomous systems, especially for multiple marine systems such AUVs, UUVs, and USVs, that rely on acoustic communication which is characterized by intermittent failures and latency [16].

The rest of the paper is organized as follows. In Section 2, preliminaries are presented and problems concerned are formulated. In Section 3, consensus of the multiagent system with single integrator, double integrator, and highorder integrator are analyzed under different situations. And simulations to prove the results are given in Section 4 . Conclusions are made in Section 5.

\section{Preliminaries and Problem Formulation}

2.1. Graph Theory. Graph theory has played an important role in analysis of multiagent systems for its advantages in modeling the interactions between agents. As graph theory has been introduced in many relative articles, only necessary notations are put forward here. Consider a system with $n$ agents and the topology graph consists of a vertex set $v=$ $\{1,2, \ldots, n\}$, an edge set $\varepsilon=\{(j, i): i, j \in v\} \subseteq v \times v$, and an adjacent matrix $A=\left[a_{i j}\right] \in R^{n \times n}$. If $\varepsilon_{j i} \in \varepsilon$, then $a_{i j}>0$ which means that agent $i$ can receive information from agent $j$; else $a_{i j}=0$. The set of neighbors of agent $i$ is denoted by $N_{i}=\left\{v_{j}:\left(v_{j}, v_{i}\right) \in \varepsilon\right\}$. A directed spanning tree is a graph that has one node called root node, which has a directed path to all of the other nodes. The Laplacian matrix $L \in R^{n \times n}$ is defined as follows:

$$
L=\left\{\begin{array}{cc}
-a_{i j}, & i \neq j ; i, j \in v \\
& \sum_{j=1, j \neq i}^{n} a_{i j} .
\end{array}\right.
$$

The following definitions are introduced for further discussion.

Definition 1 (subgraph [8]). Considering a topology graph $G=(v, \varepsilon, A)$, then $G_{1}=\left(v_{1}, \varepsilon_{1}, A_{1}\right)$ is a subgraph of $G$ if (1) $v_{1} \subseteq v$; (2) $\varepsilon_{1} \subseteq \varepsilon$.

Definition 2 (union of graphs [7]). Set the union of topology graphs $G_{k}=\left(v_{k}, \varepsilon_{k}, A_{k}\right)$ as $G=(v, \varepsilon, A)$; then, (1) $v_{k} \in v$; (2) $\varepsilon_{k} \in \varepsilon$.

2.2. Control Protocol. In this paper, system states are sampled and transmitted at discrete instants and the controller is designed based on the periodic sampling and transmitting information. For agent $i$, control input between $\left[t_{k}, t_{k+1}\right)$ can be presented as follows:

$$
u_{i}(t)=K \sum_{j \in N_{i}} a_{i j}(t)\left(x_{j}\left(t_{k}\right)-x_{i}\left(t_{k}\right)\right) \quad t_{k} \leq t<t_{k+1},
$$

where $K$ is controller gain with proper dimension to be decided, the value of $a_{i j}(t)$ should be defined as

$$
a_{i j}(t)=\left\{\begin{array}{ll}
0 & t_{k} \leq t<t_{k}+\tau_{i j}(k) \\
1 & t_{k}+\tau_{i j}(k) \leq t<t_{k+1}
\end{array} .\right.
$$

In the above equation, $\tau_{i j}(k)$ denotes the transmission delay between agent $i$ and agent $j$ during the period $\left[t_{k}, t_{k+1}\right)$. From the structure of the controller, it is obvious that the control input is not constant during $\left[t_{k}, t_{k+1}\right)$ and each agent in the system updates its input asynchronously. It is also worth noticing that (3) implies that $\tau_{i j}<t_{k+1}-t_{k}$, which is not necessarily guaranteed for communication system with random delays. So the following assumption is introduced about communication delays.

Assumption 3 (bounded transmission delays). There exist positive constant values $\tau_{\min }$ and $\tau_{i j}$ denoting the delay between agent $i$ and agent $j ; T$ presents the time period for sampling and transmitting data. The following condition is satisfied;

$$
T-\tau_{i j}(k)>\tau_{\min }, \quad \text { for any } k .
$$

From a practical point of view, it can be assumed that, for any packet with transmission delay that cannot satisfy Assumption 3, the packets are regarded as being lost.

2.3. Model. With the proposed control algorithm as in (2), the multiagent systems consist of three typical dynamics that are concerned. And the corresponding discrete-time system presentations are also presented in Table 1. 
For $m$ th high-order system, the matrices $A$ and $B$ are as follows:

$$
\begin{aligned}
A & =\left[\begin{array}{ccccc}
0 & 1 & 0 & \cdots & 0 \\
0 & 0 & 1 & \ddots & \vdots \\
0 & 0 & 0 & \ddots & 0 \\
\vdots & \vdots & \vdots & \ddots & 1 \\
0 & 0 & 0 & \cdots & 0
\end{array}\right] \in \mathbf{R}^{m \times m}, \\
B & =\left[\begin{array}{llll}
0 & \cdots & 0 & 1
\end{array}\right]^{T} \in \mathbf{R}^{m \times 1} .
\end{aligned}
$$

Definition 4 (consensus [11]). Consensus of the multiagent system is regarded as being achieved when the following equation is satisfied:

$$
\lim _{t \rightarrow \infty}\left\|x_{i}(t)-x_{j}(t)\right\|=0, \quad \forall i, j \in v .
$$

\section{Consensus of Multiagent System}

In this section, consensus problem of multiagent system consists of different agent dynamics that is discussed separately. With proper assumptions made, we have proposed the conditions needed for consensusability of the multiagent system.

\subsection{Case 1: Single Integrator Agent}

3.1.1. With Communication Delays and No Packet Losses. Define $X(t)=\left[\begin{array}{llll}x_{1}(t) & x_{2}(t) & \cdots & x_{n}(t)\end{array}\right]^{T}$ as the state of the multiagent system. Since it can be easily extended to multistate through Kronecker production, it is assumed here that each agent has only one state for briefness of description. Based on the controller as (2) and the discrete time model in Table 1, the dynamics of multiagent system can be denoted by

$$
X\left(t_{k+1}\right)=\left(I-\alpha\left(t_{0} L_{0}+t_{1} L_{1}+\cdots+t_{m} L_{m}\right)\right) X\left(t_{k}\right),
$$

where controller gain $K$ is presented as $\alpha \in R$ in this case. Because of transmission delays, Laplacian matrix is not invariant. So $L_{0}, L_{1}, \ldots, L_{m}$ represent the Laplacian matrices that exist during $\left[t_{k}, t_{k+1}\right)$ and $t_{0}, t_{1}, \ldots, t_{m}$ are the durations for corresponding Laplacian matrices satisfying $t_{0}+t_{1}+\cdots+$ $t_{m}=T$. In fact, $L_{0}$ is a Laplacian matrix with no connections between agents and since no packet losses are concerned, for any $0 \leq i \leq m-1$, the topology associated with $L_{i}$ is a subgraph of the topology associated $L_{i+1}$. To prove the consensusability of discrete-time system as in (7), following lemmas about nonnegative matrix and stochastic matrix are introduced beforehand.

Lemma 5 (see [26]). Let $M \in \mathbf{R}^{n \times n}$ be a stochastic matrix. If $M$ has an eigenvalue $\lambda=1$ with algebraic multiplicity equal to one and all of the other eigenvalues satisfy $|\lambda|<1$, then $M$ is SIA. That is, $\lim _{n \rightarrow \infty} M^{n} \rightarrow \mathbf{1} y^{T}$.

Lemma 6 (see [9]). A stochastic matrix has algebraic multiplicity equal to one for its eigenvalue $\lambda=1$ if and only if the graph associated with the matrix has a spanning tree. Furthermore, a stochastic matrix with positive diagonal elements has the property that $|\lambda|<1$ for every eigenvalue not equal to one.

Lemma 7 (see [27]). Let $S_{1}, S_{2}, \ldots, S_{k}$ be a finite set of SIA matrices with property that, for each sequence $S_{i_{1}}, S_{i_{2}}, \ldots, S_{i_{j}}$ of positive length, the matrix product $S_{i_{j}} S_{i_{j-1}} \cdots S_{i_{1}}$ is SIA. Then, for each infinite sequence $S_{i_{1}}, S_{i_{2}}, \ldots$, there exists a column vector $y$ such that $\lim _{j \rightarrow \infty} S_{i_{j}}, S_{i_{j-1}}, \ldots, S_{i_{1}}=1 y^{T}$.

Lemma 8 (Gershgorin circle criterion [28]). All eigenvalues of a matrix $E=\left[e_{i j}\right] \in R^{N \times N}$ are located within the union of $N$ discs as follows:

$$
\bigcup_{i=1}^{N}\left\{z \in C:\left|z-e_{i i}\right| \leq \sum_{j \neq i}\left|e_{i j}\right|\right\} .
$$

Lemma 9 (see [29]). Let $m \geq 2$ be a positive integer and let $P_{1}, P_{2}, \ldots, P_{m}$ be nonnegative matrices with positive diagonal elements; then, $P_{1} P_{2} \cdots P_{m} \geq \gamma\left(P_{1}+P_{2}+\cdots+P_{m}\right)$, with $\gamma>0$. And if the digraph associated with $\gamma\left(P_{1}+P_{2}+\cdots+P_{m}\right)$ has a spanning tree, the graph associated with $P_{1} P_{2} \cdots P_{m}$ also has a spanning tree.

Besides, some assumptions also need to be proposed.

Assumption 10 (quantized transmission delays). There exists a small positive value $\Delta$ such that, for all $i, j \in 1,2, \ldots, n$,

$$
\tau_{i j}(k)=q \Delta, \quad q \in 1,2, \ldots, m_{q} .
$$

Assumption 10 indicates that transmission delays can be quantized and must be a multiple of fundamental delay time $\Delta$. Assumption 10 is realizable and practical because all agents are operated under digital computers and the smaller the $\Delta$ is, the higher the accuracy is.

Assumption 11. The designed communication topology for the multiagent system has a directed spanning tree.

This assumption means that if there are no packet losses and communication delays, the topology of the multiagent system has a spanning tree at every instant $t_{k}$. Combing with Assumption 3, it can be derived that the union of digraphs associated with $L_{0}, L_{1}, \ldots, L_{m}$ has a directed spanning tree. Then based on introduced lemmas and assumptions, the consensus of the system described as in (7) can be proposed.

Theorem 12. For multiagent system consisting of single integrator dynamics, with control protocol designed as (2) and Assumptions 3-11, the consensus of the multiagent system can be reached if the controller gain is set to be $\alpha=1 / T d_{\max }$, where $d_{\max }$ is the largest in-degree of the Laplacian matrices $L_{0}, L_{1}, \ldots L_{m}$.

Proof. First of all, since there are a limited number of possible Laplacian matrices for fixed number of agents, $d_{\max }$ can be precalculated without consideration about communication 
delays; then, it can be viewed as a constant in subsequent presentation. By substituting $\alpha=1 / T d_{\text {max }}$ into (7), the system can be transformed as

$$
\begin{aligned}
X\left(t_{k+1}\right) & =\left(I-\frac{1}{d_{\max }}\left(\frac{t_{0}}{T} L_{0}+\frac{t_{1}}{T} L_{1}+\cdots+\frac{t_{m}}{T} L_{m}\right)\right) X\left(t_{k}\right) \\
& =M_{k} X\left(t_{k}\right) .
\end{aligned}
$$

Since Assumption 11 holds and there are no packet losses, $L_{m}$ has a spanning tree and the interaction graphs associated with $L_{0}, L_{1}, \ldots, L_{m-1}$ are subgraphs of the topology associated with $L_{m}$. Then, for the union of graphs $L_{0}, L_{1}, \ldots, L_{m-1}$, there is a simple eigenvalue equal to zero. According to Lemma 8 , it is not difficult to conclude that all of other eigenvalues of $\left(1 / d_{\max }\right)\left(\left(t_{0} / T\right) L_{0}+\left(t_{1} / T\right) L_{1}+\cdots+\left(t_{m} / T\right) L_{m}\right)$ are located within the circle with origin point of $(1,0)$ and radius of 1 . Thus, it can be obtained that $M_{k}$ is a matrix with simple eigenvalues equal to one and the others within the unit circle of the complex plane which means that the topology associated with $M_{k}$ has a spanning tree according to Lemma 6.

In addition, since all the nondiagonal entries of Laplacian matrices are nonpositive, the nondiagonal entries of $M_{k}$ are nonnegative. All diagonal elements of $M_{k}$ are less than one due to the choice of $\alpha$. And since Laplacian matrices $L_{0}, L_{1}, \ldots, L_{m}$ all have zero row sums, $M_{k}$ has row sum equal to one; that is, $M_{k}$ is a stochastic matrix. As a conclusion, $M_{k}$ is a stochastic nonnegative matrix with positive diagonal elements and it can be derived from Lemma 5 that $M_{k}$ is SIA.

Because $M_{k}$ has a spanning tree, $M_{k} M_{k+1} \cdots M_{k+l}$ also has a spanning tree according to Lemma 9. In addition, the stochastic matrices with positive diagonal entries are closed under matrix multiplication, so that $M_{k} M_{k+1} \cdots M_{k+l}$ is also a stochastic matrix with positive diagonal elements. According to Lemmas 6 and 5, the matrix $M_{k} M_{k+1} \cdots M_{k+l}$ is SIA.

With Assumption 10, it can be derived that there is a finite number of $M_{k}$. After that, Lemma 7 can be applied to acquire that the multiagent system as in (10) can reach a consensus. Theorem 12 is proved.

3.1.2. With Packet Losses and Communication Delays. In the above section, consensus problem with communication delays has been solved that lay firm foundation for the problem concerned in the situation where packet losses must be taken into consideration. In this case, Assumption 3 is no longer satisfied. As mentioned previously, Assumption 3 is a relative strict condition to be fulfilled in practice. So the following assumption is proposed in addition.

Assumption 13. Set the success ratio of transmission between two agents as $p \in(0,1), \forall \mu \in(0,1)$; there exists an integer $l$ that satisfies $1-(1-p)^{l}>\mu$. If $\mu$ is chosen to be close to 1 enough, it is reasonable to assume that the transmission can be successful for at least one time during $l$ periods. Besides, under Assumption 13, the communication delay can still satisfy Assumption 3 for the successful transmitted information. With Assumption 11, this assumption essentially means that the union of digraphs within $l$ periods has a directed spanning tree.

Theorem 14. For multiagent system consisting of single integrator dynamics satisfying Assumptions 10-13, with control protocol designed as in (2), the consensus of the multiagent system can be reached with the same controller gain $\alpha$ adopted as in Theorem 12.

Proof. In this situation, since Assumption 3 is no longer satisfied all the time, $L_{m}$ cannot always have a spanning tree. However, according to Assumptions 11 and 13, the digraph associated with $M_{k}+M_{k+1}+\cdots+M_{k+l-1}$ will have a spanning tree. Since $M_{k}$ is still a stochastic nonnegative matrix with positive diagonal elements, Lemma 9 still holds; that is, the relation $M_{k} M_{k+1} \cdots M_{k+l-1} \geq \gamma\left(M_{k}+M_{k+1}+\cdots+M_{k+l-1}\right)$ exists. Thus, define $Q_{k, l}=M_{k} M_{k+1} \cdots M_{k+l-1}$ such that

$$
X\left(t_{k+l}\right)=M_{k} M_{k+1} \cdots M_{k+l-1} X\left(t_{k}\right)=Q_{k, l} X\left(t_{k}\right) \text {. }
$$

Since $Q_{k, l}$ is a stochastic nonnegative matrix with positive diagonal elements and the associated graph has a spanning tree, the problem regarding packet losses and communication delays can be handled in a similar way as in proof of Theorem 12 . So, the consensusability of multiagent system with packet losses and communication delays is guaranteed.

Remark 15. Based on the analysis of this section, it can be found that Theorem 12 can be viewed as a special case of Theorem 14 with $l=1$. With the proposed control protocol and proper choice of controller gain, the multiagent system can reach consensus as long as the union of the digraphs within finite periods has a spanning tree.

\subsection{Case 2: Double Integrator Agent}

3.2.1. With Communication Delays and No Packet Losses. For second order multiagent system, the corresponding variables are defined as $X(t)=\left[\begin{array}{llll}x_{1}(t) & x_{2}(t) & \cdots & x_{n}(t)\end{array}\right]^{T}, V(t)=$ $\left[\begin{array}{llll}v_{1}(t) & v_{2}(t) & \cdots & v_{n}(t)\end{array}\right]^{T}$, respectively. Then based on the discrete time model of second order system in Table 1, it can be obtained that for agent $i$

$$
\begin{array}{r}
v_{i}\left(t_{k+1}\right)=v_{i}\left(t_{k}\right)+\sum_{j \in N_{i}, j=0}\left(T-\tau_{i j}(k)\right) u_{i j}(k), \\
x_{i}\left(t_{k+1}\right)=x_{i}\left(t_{k}\right)+T v_{i}\left(t_{k}\right) \\
+\sum_{j \in N_{i}, j=0} \frac{\left(T-\tau_{i j}(k)\right)^{2}}{2} u_{i j}(k),
\end{array}
$$

where $u_{i j}(k)$ is the control input results from the relative state information between agent $i$ and agent $j . u_{i 0}$ denotes the default control value of agent $i$ that is usually set as 0 and $\tau_{i 0}=0$ since the input is default.

To make (13) more concise, some transformations need to be carried out. Set $u_{i j}(k)=\left(2 /\left(T-\tau_{i j}(k)\right)^{2}\right) u_{i j}^{\prime}(k)$. The transformation process is applicable because, during the 
period $\left[t_{k}, t_{k+1}\right), \tau_{i j}(k)$ are known to the agent $i$. Equations (12) and (13) are revised as

$$
\begin{aligned}
& v_{i}\left(t_{k+1}\right)=v_{i}\left(t_{k}\right)+\sum_{j \in N_{i}, j=0} \frac{2}{\left(t_{k+1}-\tau_{i j}\right)} u_{i j}^{\prime}, \\
& x_{i}\left(t_{k+1}\right)=x_{i}\left(t_{k}\right)+T v_{i}\left(t_{k}\right)+\sum_{j \in N_{i}, j=0} u_{i j}^{\prime} .
\end{aligned}
$$

With the controller gain chosen as $K=\left[\begin{array}{ll}\alpha & \beta\end{array}\right], u_{i j}^{\prime}(k)=$ $\alpha\left(x_{j}\left(t_{k}\right)-x_{i}(k)\right)+\beta\left(v_{j}\left(t_{k}\right)-v_{i}(k)\right)$. The dynamics of the multiagent system can be presented as

$$
\left[\begin{array}{l}
X\left(t_{k+1}\right) \\
V\left(t_{k+1}\right)
\end{array}\right]=\left[\begin{array}{cc}
I_{n}-\alpha L_{k} & T I_{n}-\beta L_{k} \\
-\alpha L_{k}^{\prime} & I_{n}-\beta L_{k}^{\prime}
\end{array}\right]\left[\begin{array}{l}
X\left(t_{k}\right) \\
V\left(t_{k}\right)
\end{array}\right]
$$

As we know, topology associated with $L_{k}$ will have a spanning tree if Assumption 11 holds. $L_{k}^{\prime}$ has the same structure as $L_{k}$ except for all its items that have been multiplied by $2 /\left(T-\tau_{i j}(k)\right)$. Thus, topology of $L_{k}^{\prime}$ also has a spanning tree. The consensus problem for system depicted as (15) can be discussed in a similar way as in Cao and Ren [8].

Lemma 16 (Cao and Ren [8]). With the decomposition of system transfer matrix as in (16), since $L_{k}, L_{k}^{\prime}$ all have spanning tree, if the conditions (1) and (2) are satisfied, consensus of the system can be reached

$$
\begin{aligned}
& {\left[\begin{array}{cc}
I_{n}-\alpha L_{k} & T I_{n}-\beta L_{k} \\
-\alpha L_{k}^{\prime} & I_{n}-\beta L_{k}^{\prime}
\end{array}\right]} \\
& \quad=\left[\begin{array}{cc}
I_{n}-T I_{n}-\alpha L_{k} & T I_{n}-\beta L_{k} \\
T I_{n}-\alpha L_{k}^{\prime} & I_{n}-T I_{n}-\beta L_{k}^{\prime}
\end{array}\right]+\left[\begin{array}{cc}
T I_{n} & \mathbf{0} \\
-T I_{n} & T I_{n}
\end{array}\right] .
\end{aligned}
$$

(1) $I_{n}-T I_{n}-\alpha L_{k},(1-T) I_{n}-\beta L_{k}^{\prime}$ are nonnegative matrices with positive diagonal entries and $T I_{n}-\beta L_{k}, T I_{n}-\alpha L_{k}^{\prime}$ are nonnegative matrices.

(2) The infinite norm of matrix $\left[\begin{array}{cc}T I_{n} & 0 \\ -T I_{n} & T I_{n}\end{array}\right]$ is less than 1.

The first condition is aimed to guarantee that the first matrix is a nonnegative one with positive diagonal entries. In addition, it is not difficult to find that the matrix is also stochastic. Then the matrix is SIA and Lemma 7 is applicable. And the second condition is to make sure that multiplication of infinite number of the second matrix goes to zero. More detailed proofs can be found in Cao and Ren [8].
Theorem 17. For multiagent system consisting of double integrator agents, with Assumptions 3-11 holding, if the sampling and transmitting period $T$ and controller gain $K$ can satisfy the following conditions, the consensus of the multiagent system is guaranteed:

$$
\begin{aligned}
& \text { (1) } 1 / 4<T<1 / 2 ; \\
& \text { (2) } \alpha=\beta=\tau_{\min } / 4 d_{\max } \text {. }
\end{aligned}
$$

Proof. If condition (1) is satisfied, the infinite norm of matrix $\left[\begin{array}{cc}T I_{n} & 0 \\ -T I_{n} & T I_{n}\end{array}\right]$ is less than 1. Define $d_{\max }, d_{\max }^{\prime}$ that represent the largest in-degree for $L_{k}, L_{k}^{\prime}$, respectively. $d_{\max }^{\prime}$ is random due to the randomicity of transmission delays. From Assumption 3 , it is obvious that

$$
\tau_{\min } d_{\max }^{\prime}<d_{\max }
$$

Then, with the second condition, it can be acquired that

$$
\sup \left(\frac{\tau_{\min }}{4 d_{\max }} d_{\max }^{\prime}\right)<\frac{1}{4} .
$$

As a result, $T-\alpha d_{\max }^{\prime}>0$. Besides, it is not difficult to find that $1-T-\beta d_{\max }^{\prime}>0$ since $\tau_{\min }<T$. As a result, it can be concluded that if the conditions are satisfied, Lemma 16 is also fulfilled and the consensusability of the multiagent system with double integrator dynamics is proved.

3.2.2. With Communication Delays and Packet Losses. For second order system with packet losses, Assumption 11 cannot hold and the Laplacian matrices $L_{k}, L_{k}^{\prime}$ discussed in the above section no longer have a spanning tree all the time. In this situation, Lemma 16 cannot directly be applied. Inspired by Qin et al. [17], a covariable can be introduced here with definition as

$$
y_{i}\left(t_{k}\right)=a v_{i}\left(t_{k}\right)+b x_{i}\left(t_{k}\right)
$$

where $a, b$ are constant parameters. It is easily derived that if $x\left(t_{k}\right)$ and $y\left(t_{k}\right)$ can reach consensus, the velocity of the system will also achieve consensus. So in the following discussion, the consensus problem of system states $x\left(t_{k}\right)$ and $y\left(t_{k}\right)$ is concerned. Besides, default control of agent $i$ also needs to be set as $u_{i 0}^{\prime}=-c v_{i}\left(t_{k}\right), i \in\{1,2, \ldots, n\}$.

As a result, the multiagent system can be transformed into

$$
\left[\begin{array}{l}
x\left(t_{k+1}\right) \\
y\left(t_{k+1}\right)
\end{array}\right]=\left[\begin{array}{cc}
\left(1-\frac{b T}{a}(1-c T)\right) I_{n}-\left(\alpha-\frac{b \beta}{a}\right) L_{k} & \frac{T}{a}(1-c T) I_{n}-\frac{\beta}{a} L_{k} \\
\left(c b T-\frac{b^{2} T}{a}(1-c T)\right) & I_{n}-\left(\alpha-\frac{b \beta}{a}\right) L_{k}^{\prime}\left(\frac{b T}{a}(1-c T)+1-c T\right) I_{n}-\frac{\beta}{a} L_{k}^{\prime}
\end{array}\right]\left[\begin{array}{c}
x\left(t_{k}\right) \\
y\left(t_{k}\right)
\end{array}\right]
$$

With proper choices of parameters $\alpha, \beta, a, b$, and $T$, the system matrix can be a nonnegative stochastic matrix with positive diagonal elements. In fact, to ensure that the transfer matrix is stochastic, $b \equiv 1$. Then if Assumption 13 holds, the 
system described as (20) can reach a consensus with similar proof as Theorem 14. However, because of the existence of default control input, the consensus value of system velocity is zero which is usually the case for rendezvous problem not for formation control or flocking. So in order to obtain the consensus for multiagent system without adding the control input $u_{i 0}^{\prime}$, in the subsequence of this paper, a different assumption about interaction topology needs to be made.

Assumption 18. The designed communication topology for the multiagent system is a directed spanning tree. Similarly, with Assumption 13, this assumption essentially means that the union of digraphs within $l$ periods is a directed spanning tree.

Consider two agents $i, j$ and assume that there is a directed link from agent $i$ to $j$. According to Assumption 13, there exist a positive integer $g$ with $1 \leq g \leq l$ such that the data transmission is successful during the period $\left[t_{k+g-1}, t_{k+g}\right)$ and packets dropout during the previous $g-1$ periods. Define the delay during $\left[t_{k+g-1}, t_{k+g}\right)$ as $\tau_{i j}\left(t_{k}^{g}\right)$ which satisfies Assumption 3. Adopt the controller gain as $\alpha_{j}\left(t_{k}^{g}\right)=$ $\beta_{j}\left(t_{k}^{g}\right)=\left(T-\tau_{i j}\left(t_{k}^{g}\right)\right) / 4$ and the following dynamics can be obtained for agents $i$ and $j$ :

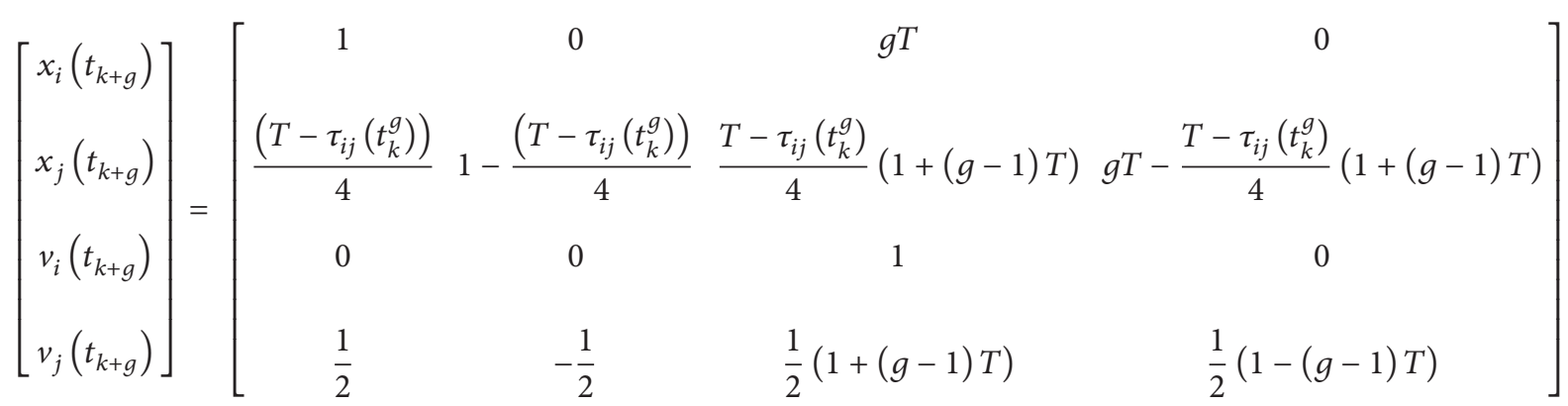

$$
\begin{aligned}
& \times\left[\begin{array}{l}
x_{i}\left(t_{k}\right) \\
x_{j}\left(t_{k}\right) \\
v_{i}\left(t_{k}\right) \\
v_{j}\left(t_{k}\right)
\end{array}\right] \text {. }
\end{aligned}
$$

Define the states error between agents $i$ and $j$ at time instants as $t_{k}$ and $t_{k+g}$ as follows:

$$
\begin{aligned}
& \zeta_{i j}(d+1)=x_{j}\left(t_{k+g}\right)-x_{i}\left(t_{k+g}\right), \\
& \xi_{i j}(d+1)=v_{j}\left(t_{k+g}\right)-v_{i}\left(t_{k+g}\right),
\end{aligned}
$$

$$
\begin{aligned}
& \zeta_{i j}(d)=x_{j}\left(t_{k}\right)-x_{i}\left(t_{k}\right), \\
& \xi_{i j}(d)=v_{j}\left(t_{k}\right)-v_{i}\left(t_{k}\right),
\end{aligned}
$$

where $d$ represents the number of successful transmissions. Then the following equation is acquired denoting the error dynamics between agents $i$ and $j$ :

$$
\left[\begin{array}{l}
\zeta_{i j}(d+1) \\
\xi_{i j}(d+1)
\end{array}\right]=\left[\begin{array}{c}
\left(1-\frac{\left(T-\tau_{i j}\left(t_{k}^{g}\right)\right)}{4}\right)\left(g T-\frac{T-\tau_{i j}\left(t_{k}^{g}\right)}{4}(1+(g-1) T)\right) \\
-\frac{1}{2}
\end{array}\right]\left[\begin{array}{l}
\zeta_{i j}(d) \\
\xi_{i j}(d)
\end{array}\right] .
$$

According to the error dynamics depicted as above, the following lemma can be proposed.

Theorem 19. For multiagent system consisting of double integrator dynamics as in Table 1, with Assumptions 13 and 18, the consensusability of the system can be guaranteed with packet losses and transmission delays as long as the discrete error dynamics as in (23) is stable.

Proof. Without loss of generality, set agent $i$ as the root node of the directed spanning tree. Define $N_{i}^{0}$ as the set of agents that receive information from agent $i$. If the error dynamics as 
TABLE 1: Continuous time and discrete time presentations of agent dynamics.

\begin{tabular}{lcc}
\hline Agent type & Continuous time system & Discrete time presentation \\
\hline Single integrator & $\dot{x}_{i}(t)=u_{i}(t)$ & $x_{i}\left(t_{k+1}\right)=x_{i}\left(t_{k}\right)+T K \sum_{j \in N_{i}} a_{i j}\left(x_{j}\left(t_{k}\right)-x_{i}\left(t_{k}\right)\right)$ \\
\hline Double integrator & $\dot{x}_{i}(t)=v_{i}(t)$ & $x_{i}\left(t_{k+1}\right)=x_{i}\left(t_{k}\right)+T v_{i}\left(t_{k}\right)+\frac{T^{2}}{2} K \sum_{j \in N_{i}} a_{i j}\left(x_{j}\left(t_{k}\right)-x_{i}\left(t_{k}\right)\right)$ \\
& $\dot{v}_{i}(t)=u_{i}(t)$ & $v_{i}\left(t_{k+1}\right)=v_{i}\left(t_{k}\right)+T K \sum_{j \in N_{i}} a_{i j}\left(x_{j}\left(t_{k}\right)-x_{i}\left(t_{k}\right)\right)$ \\
\hline High-order integrator & $\dot{x}_{i}(t)=A x_{i}(t)+B u_{i}(t)$ & $x_{i}\left(t_{k+1}\right)=e^{A T} x_{i}\left(t_{k}\right)+\int_{0}^{T} e^{A t} B K \sum_{j \in N_{i}} a_{i j}\left(x_{j}\left(t_{k}\right)-x_{i}\left(t_{k}\right)\right) d t$ \\
\hline
\end{tabular}

in (23) is stable, that is, $\lim _{d \rightarrow \infty} \zeta_{i j}(d)=0, \lim _{d \rightarrow \infty} \xi_{i j}(d)=$ 0 . It is obvious that agents $i$ and $j$ can reach a consensus for any $j \in N_{i}^{0}$ with finite time periods since Assumption 18 holds.

Then there exist a positive number $m_{j}$ and a time instant $t_{m_{j}}$ such that $\left[\zeta_{i j}\left(m_{j}\right) \quad \xi_{i j}\left(m_{j}\right)\right]^{T}=\left[\begin{array}{ll}0 & 0\end{array}\right]^{T}$ for $t>t_{m_{j}}$. With definition of $t_{m}=\max \left\{t_{m_{j}}\right\}, j \in N_{i}^{0}$, it can be derived that when $t>t_{m}, x_{j}(t)=x_{i}(t), v_{j}(t)=v_{i}(t)$, for all $j \in N_{i}^{0}$ which means that the consensus has been reached between agents $i$ and $N_{i}^{0}$. Since the discrete error dynamics is as in
(23) set no restrictions on initial states of agents and the agents belonging to $N_{j}^{0}$ will reach consensus with agent $j$ in a similar way. Finally, the whole multiagent system will reach a consensus.

To be more illustrative, the process can be shown in Figure 1. The error dynamics between different agents in the dash line circle can be presented as (23). The agents in the real line circle mean that the consensus has been reached among them.

In the following, an algorithm to testify the Schur stability of discrete-time dynamics system as in (23) is proposed. Since all communication delay $\tau_{i j}$ is random, the transfer matrix

$$
M=\left[\begin{array}{c}
\left(1-\frac{T-\tau_{i j}\left(t_{k}^{g}\right)}{4}\right) \\
-\frac{1}{2}
\end{array}\right]
$$

can be viewed as an interval matrix $\left[M^{m}, M^{M}\right]=\{M=$ $\left.\left[m_{i j}\right]: m_{i j}^{m}<m_{i j}<m_{i j}^{M}, i, j=1,2\right\}$ for fixed $g$ with the expressions of $M^{m}$ and $M^{M}$ as follows:

$$
\begin{gathered}
M^{m}=\left[\begin{array}{cc}
1-\frac{T}{4} & g T-\frac{T}{4}(1+(g-1) T) \\
-\frac{1}{2} & \frac{1}{2}(1-(g-1) T)
\end{array}\right] ; \\
M^{M}=\left[\begin{array}{cc}
1-\frac{\tau_{\min }}{4} & \left.g T-\frac{\tau_{\min }}{4}(1+(g-1) T)\right) \\
-\frac{1}{2} & \frac{1}{2}(1-(g-1) T)
\end{array}\right] .
\end{gathered}
$$

The following lemma needs to be introduced concerning the stability of interval matrix.

Lemma 20. An interval matrix $\left[R^{m}, R^{M}\right]$ is Schur stable if and only if there are finitely subinterval matrices $\left[R_{i}^{m}, R_{i}^{M}\right] \subset$ $\left[R^{m}, R^{M}\right], 1 \leq i \leq k$, such that

$$
\left[R^{m}, R^{M}\right]=\bigcup_{i=1}^{k}\left[R_{i}^{m}, R_{i}^{M}\right] .
$$

And for each $i, 1 \leq i \leq k,\left[R_{i}^{m}, R_{i}^{M}\right]$ satisfies the following conditions.
(1) $R_{i 0}$ is Schur stable and there exists a positive definite matrix $P=P^{T}$ satisfying

$$
R_{i 0}{ }^{T} P_{i} R_{i 0}-P_{i}+I=0 .
$$

(2) $\phi\left(\Delta R_{i}\right)<\left[\phi\left(R_{i 0}\right)^{2}+1 /\left|P_{i}\right|_{\infty}\right]^{1 / 2}-\phi\left(R_{i 0}\right)$. $\phi(\cdot)$ is an operator $\phi(R)=\max \left\{|R|_{1},|R|_{\infty}\right\}$ and the definitions of $\Delta R_{i}$ and $R_{i 0}$ are as follows:

$$
\begin{aligned}
\Delta R_{i} & =\frac{1}{2}\left(R_{i}^{M}-R_{i}^{m}\right), \\
R_{i 0} & =\frac{1}{2}\left(R_{i}^{M}+R_{i}^{m}\right) .
\end{aligned}
$$

(3) Subinterval matrices $\bigcup_{i=1}^{k}\left[R_{i}^{m}, R_{i}^{M}\right]$ are complete decomposition of interval matrix $\left[R^{m}, R^{M}\right]$ which means that $\forall R \in\left[R^{m}, R^{M}\right], \exists i \in[1,2, \ldots, k]$ such that $R \in\left[R_{i}^{m}, R_{i}^{M}\right]$.

Proof. See Wang et al. [30] and Liao et al. [31] for details.

Consider the parameters in (23), according to Assumption 3 and $\tau_{\min }$ actually denotes the smallest processing 


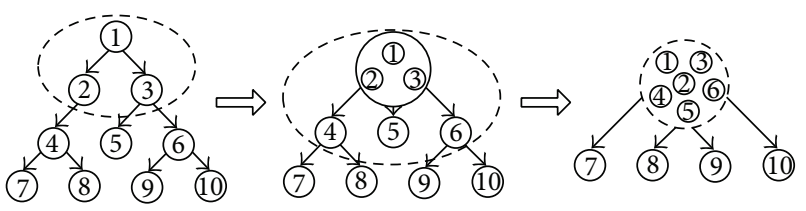

FIGURE 1: Consensus process of multiagent system under directed spanning tree.

time available for the embedded system. $T$ is the sampling and transmitting period for the multiagent system and for acoustic communication system it also indicates the largest distance available between two linked agents since the relation Dis $<v_{c}\left(T-\tau_{\text {min }}\right)$ has to be satisfied, where $v_{c}$ is the acoustic velocity. Due to the physical meanings of $\tau_{\min }, T$, they usually are predetermined once the multiagent system structure is determined. As for $g$, according to Assumption 13 and the derivation of (23), $g$ is a time varying positive integrator variable satisfying $1 \leq g \leq l$. So the following lemma is put forward.

Lemma 21. The system transfer matrix in (23) is Schur stable if, for every fixed $g \in[1,2, \ldots, l],\left[M^{m}, M^{M}\right]$ is Schur stable.

Proof. Define the interval matrix $\left[M^{m}, M^{M}\right]$ as $\left[M_{j}^{m}, M_{j}^{M}\right]$ when $g=j, 1 \leq j \leq l$. If $\left[M_{j}^{m}, M_{j}^{M}\right]$ is Schur stable, then there exist a finite number of subinterval matrices $\left[M_{j}^{m}, M_{j}^{M}\right]=$ $\bigcup_{i=1}^{k}\left[M_{i j}^{m}, M_{i j}^{M}\right]$ satisfying conditions in Lemma 20 . In addition, the system matrix in (23) can be completely decomposed as

$$
\left[M^{m}, M^{M}\right]=\bigcup_{g=1}^{l}\left[M_{j}^{m}, M_{j}^{M}\right] .
$$

Then it can be derived that

$$
\begin{aligned}
{\left[M^{m}, M^{M}\right]=} & \left\{\bigcup_{i=1}^{k}\left[M_{i 1}^{m}, M_{i 1}^{M}\right]\right\} \cup\left\{\bigcup_{i=1}^{k}\left[M_{i 2}^{m}, M_{i 2}^{M}\right]\right\} \\
& \cup \cdots \cup\left\{\bigcup_{i=1}^{k}\left[M_{i l}^{m}, M_{i l}^{M}\right]\right\} .
\end{aligned}
$$

The above equation means that the system matrix can be decomposed into finite number of subinterval matrices that all satisfy the conditions in Lemma 20. The matrix is Schur stable.

Even though Lemmas 20 and 21 have provided conditions to guarantee the Schur stability of the discrete interval system matrix, method to completely decompose an interval matrix is needed. Based on the definition of $R_{i 0}$ and $\Delta R_{i}$ in (28), the interval matrix $\left[R^{m}, R^{M}\right]$ can be presented as $\left[R_{0} \pm \Delta R\right]$. With this presentation, it is natural to adopt the decomposition as $\left[R^{m}, R^{M}\right]=\left[R_{0}-\Delta R, R_{0}\right] \cup\left[R_{0}, R_{0}+\right.$ $\Delta R]$. However, the decomposition is not always complete. Denote the element of the interval matrix at $i$ th row and $j$ th column by $\left[R^{m}(i, j), R^{M}(i, j)\right]$. Then divide the interval into two subintervals as follows:

$$
\left[R^{m}(i, j), R^{M}(i, j)\right]=R_{L}(i, j) \cup R_{H}(i, j)
$$

with $R_{L}(i, j)$ and $R_{H}(i, j)$ defined as

$$
\begin{aligned}
& R_{L}(i, j)=\left[R_{0}(i, j)-\Delta R(i, j), R_{0}(i, j)\right], \\
& R_{H}(i, j)=\left[R_{0}(i, j), R_{0}(i, j)+\Delta R(i, j)\right]
\end{aligned}
$$

If $R^{m}, R^{M} \in \mathbf{R}^{n \times n}$, the interval matrix can be decomposed as follows:

$$
\left[R^{m}, R^{M}\right]=\bigcup_{k=1}^{2^{n \times n}} R_{\mathrm{inv}}(k) .
$$

Each element in $R_{\text {inv }}(k)$ at $i$ th row and $j$ th column will be either $R_{L}(i, j)$ or $R_{H}(i, j)$. Then, it is obvious that the decomposition is complete. Now, Algorithm 1 is proposed in pseudocode to testify the Schur stability of the interval matrix. If a limited number of subintervals can be obtained for each $\left[M_{j}^{m}, M_{j}^{M}\right], 1 \leq j \leq l$ that satisfy the Schur stability conditions in Lemma 20, the system matrix in (23) is stable according to Lemma 21 . That is, the multiagent system consists of second order agent with communication delays and packet losses can reach a consensus.

Through Algorithm 1, the consensusability of the multiagent system concerned can be testified. However, since the controller gain and the system parameters are predefined, no analytical solutions are provided by this method for multiagent system that could not reach consensus. Besides, the computational burden of the algorithm will increase exponentially with the order of agents. Therefore, in the next section, a theorem will be proposed to design the controller gain and solve the consensus problem of multiagent system with high-order integrator agents.

3.3. High-Order Integrator Agent. Assume that Assumption 18 still holds; similar result can be obtained as in Theorem 19; that is, if the root agent $i$ and agent $j, j \in N_{i}^{0}$, can reach consensus, the multiagent system can reach consensus. So for the high-order integrator dynamics introduced in Table 1, with communication delays, the discrete system dynamics for agent $i$ and agent $j$ can be presented as

$$
\begin{gathered}
X_{i}\left(t_{k+1}\right)=e^{A T} X_{i}\left(t_{k}\right), \\
X_{j}\left(t_{k+1}\right)=e^{A T} X_{j}\left(t_{k}\right)+\int_{o}^{T-\tau_{i j}\left(t_{k}\right)} e^{A t} d t \\
\cdot B K\left(X_{i}\left(t_{k}\right)-X_{j}\left(t_{k}\right)\right)
\end{gathered}
$$

with $K=\left[\begin{array}{llll}k_{1} & k_{2} & \cdots & k_{m}\end{array}\right]$ as the controller gain to be designed; $\tau_{i j}\left(t_{k}\right)$ is the communication delay satisfying Assumption 3. Let the states error between agent $i$ and agent $j$ at time $t_{k}$

$$
\delta\left(t_{k}\right)=X_{i}\left(t_{k}\right)-X_{j}\left(t_{k}\right)
$$




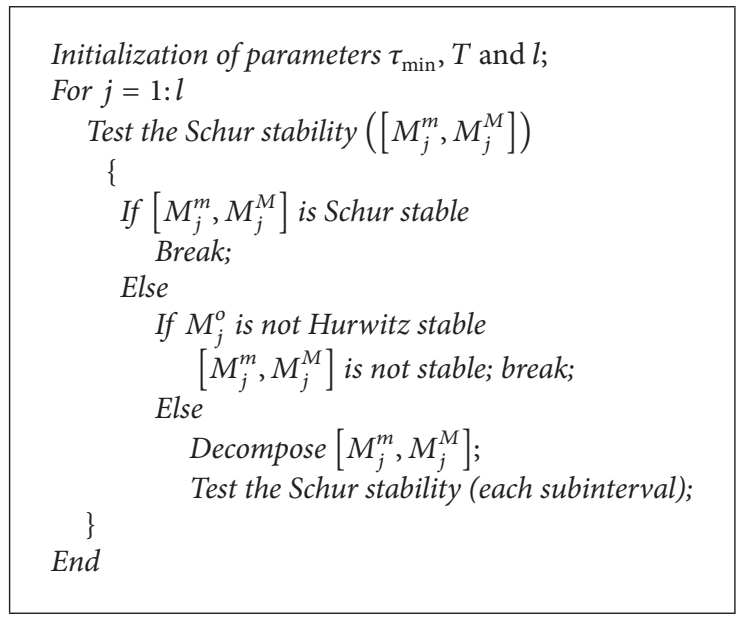

Algorithm 1

The error dynamics between two agents is

$$
\delta\left(t_{k+1}\right)=\left(e^{A T}-\int_{o}^{T-\tau_{i j}\left(t_{k}\right)} e^{A t} d t \cdot B K\right) \delta\left(t_{k}\right)
$$

Considering packet losses and according to Assumption 13 , there exists an integer $g \in[1, \ldots, l]$ such that the error dynamics during the period $\left[t_{k}, t_{k+g}\right)$ can be presented as

$$
\delta\left(t_{k+g}\right)=e^{(g-1) A T}\left(e^{A T}-\int_{o}^{T-\tau_{i j}\left(t_{k}^{g}\right)} e^{A t} d t \cdot B K\right) \delta\left(t_{k}\right) .
$$

Algorithm 1 can still be applied to test the Schur stability of system matrix equation (36) and (37). Except for the computational complexity increases exponentially with the system order, the controller gain is also difficult to design comparing with second order multiagent system. So in order to circumvent those difficulties, the following lemmas are applied to achieve the controller gain.

Lemma 22 (see [32]). For discrete time interval system as in (38), $M \in \mathbf{R}^{n \times n}, N \in \mathbf{R}^{n \times p}$, the system is stabilizable if and only if there exist a matrix $G \in \mathbf{R}^{p \times n}$ and a symmetric positive definite matrix $H \in \mathbf{R}^{n \times n}$ and scalars $\lambda_{i j}>0, i, j=$ $1,2, \ldots, n, \delta_{i j}>0, i=1,2, \ldots, n, j=1,2, \ldots, p$, satisfying (39). And the controller gain can be adopted as $K=G H^{-1}$. Consider

$$
\begin{gathered}
\delta(d+1)=\left[M_{\min }, M_{\max }\right] \delta(d)+\left[N_{\min }, N_{\max }\right] u(d), \\
u(d)=K \delta(d), \\
{\left[\begin{array}{cccc}
-H & H M_{o}^{T}+G N_{o}^{T} & H_{d} & G_{d} \\
M_{0} H+N_{0} G & -H+\Sigma & 0 & 0 \\
H_{d}^{T} & 0 & -\Lambda & \\
G_{d}^{T} & 0 & & -\Delta
\end{array}\right]<0,}
\end{gathered}
$$

where $\sum=\sum_{i, j=1}^{n} \lambda_{i j}\left|\Delta m_{i j}\right|^{2} e_{i} e_{i}^{T}+\sum_{i=1}^{n} \sum_{j=1}^{p} \delta_{i j}\left|\Delta n_{i j}\right|^{2} e_{i} e_{i}^{T}, e_{i}$ is a column vector with ith element being 1 . And

$$
\begin{gathered}
H_{d}=\underbrace{[H, \ldots, H]}_{n}, \quad G_{d}=\underbrace{\left[G^{T}, \ldots, G^{T}\right]}_{n}, \\
\Lambda=\operatorname{diag}\left\{\lambda_{11}, \lambda_{12}, \ldots, \lambda_{1 n}, \ldots, \lambda_{n 1}, \lambda_{n 2}, \ldots, \lambda_{n n}\right\}, \\
\Delta=\operatorname{diag}\left\{\delta_{11}, \delta_{12}, \ldots, \delta_{1 p}, \ldots, \delta_{n 1}, \delta_{n 2}, \ldots, \delta_{n p}\right\} .
\end{gathered}
$$

Lemma 23. The interval system matrices $\left[M_{\min }, M_{\max }\right]$ and $\left[N_{\min }, N_{\max }\right]$ are stabilizable if there are a finite number of subintervals $\left[M_{\min }^{i}, M_{\max }^{i}\right],\left[N_{\min }^{i}, N_{\max }^{i}\right], 1 \leq i \leq k$ such that, for each $i \in\{1,2, \ldots, k\}$, the system matrices $\left[M_{\min }^{i}, M_{\max }^{i}\right],\left[N_{\min }^{i}, N_{\max }^{i}\right]$ are stabilizable. And the interval system is completely decomposed which means that, at any time instant $t$, if $N(t) \in\left[N_{\min }^{i}, N_{\max }^{i}\right]$, then $M(t) \in\left[M_{\min }^{i}, M_{\max }^{i}\right]$, $1 \leq i \leq k$. And the complete decomposition can be denoted by

$$
\begin{aligned}
& {\left[M_{\min }, M_{\max }\right]=\bigcup_{i=1}^{k}\left[M_{\min }^{i}, M_{\max }^{i}\right],} \\
& {\left[N_{\min }, N_{\max }\right]=\bigcup_{i=1}^{k}\left[N_{\min }^{i}, N_{\max }^{i}\right] .}
\end{aligned}
$$

Proof. Since the system matrices $\left[M_{\min }^{i}, M_{\max }^{i}\right],\left[N_{\min }^{i}, N_{\max }^{i}\right]$ are stabilizable, suppose that the controller gain is denoted by $K$; then, it can be derived that $\left[M_{\min }^{i}, M_{\max }^{i}\right]+\left[N_{\min }^{i}, N_{\max }^{i}\right] K$ is Schur stable. Because the interval system is completely decomposed, the following decomposition is also complete

$$
\begin{aligned}
& {\left[M_{\min }, M_{\max }\right]+\left[N_{\min }, N_{\max }\right] K} \\
& \quad=\bigcup_{i=1}^{k}\left(\left[M_{\min }^{i}, M_{\max }^{i}\right]+\left[N_{\min }^{i}, N_{\max }^{i}\right] K\right) .
\end{aligned}
$$

Then according to Lemma 21, the Schur stability of $\left[M_{\min }^{i}, M_{\max }^{i}\right]+\left[N_{\min }^{i}, N_{\max }^{i}\right] K, i \in\{1,2, \ldots, k\}$ indicated Schur stability of the multiagent system. 
Comparing (36) with (37), it can be concluded that (36) is the special case of (37) when $g=1$. Actually, the interval matrices in (37) are piecewise interval, and the system can be completely decomposed into $l$ subinterval systems easily. The subinterval systems can be presented as follows:

$$
\begin{aligned}
\delta\left(t_{k+g}\right)= & e^{g A T} \delta\left(t_{k}\right)-e^{(g-1) A T} \int_{o}^{T-\tau_{i j}\left(t_{k}^{g}\right)} e^{A t} B d t \cdot u\left(t_{k}\right) \\
= & M_{g} \delta\left(t_{k}\right)+N_{g} u\left(t_{k}\right) \\
& \quad \text { for } g \in\{1,2, \ldots, l\} .
\end{aligned}
$$

Theorem 24. For multiagent system consisting of high order agents, under Assumptions 13 and 18, the consensusability of the system can be guaranteed with transmission delays and packet losses if there exists a controller gain $K$ such that the conditions in Lemma 22 can be satisfied for each $M_{g}+N_{g} K$, $g \in\{1,2, \ldots, l\}$.

Proof. If the conditions in Lemma 22 are satisfied, $M_{g}+N_{g} K$ is Schur stable. From (43), the interval system in (37) can be completely decomposed by $M_{g}$ and $N_{g}, g \in\{1,2, \ldots, l\}$. Then according to Lemma 23, the theorem is established.

In fact, the existence of controller gain $K$ is important in the above lemma. According to (39) in Lemma 22, matrix $K$ can be obtained by solving the LMI with matlab. In order to guarantee the conditions in Lemma 23, $l$ LMIs should be solved simultaneously to calculate the feedback gain $K$. Besides, it should be noticed that Theorem 24 is a general solution for controller gain of multiagent system and can be applied to all kinds of agent dynamics including single integrator, second order integrator, and general linear system.

\section{Simulations}

In this section, simulation results will be presented to demonstrate the theoretical results in Section 3. A multiagent system consists of five agents with the designed interaction topology as in Figure 2 that is concerned. The success ratio $p$ in Assumption 13 is set as 0.8 according to the property of ordinary acoustic communication equipment; if $\mu$ is chosen to be 0.999 , then $l$ can be set as 5 which means that the transmission will be successful at least one time during five periods. $\tau_{\min }$ is set to be 0.1 , which is long enough for system to calculate the system inputs. Sampling and transmitting period $T=0.5$. With all above initialization, single integrator, second order, and high-order agents are discussed separately and consensusability for every typical multiagent system is testified with packet losses and communication delays.

4.1. Single Integrator Agent. In this case, the initial state of each agent in the multiagent system is chosen randomly from 0 to $50, \alpha=2$. Simulation result is shown in Figure 3. It can be concluded that, for single integrator system, the position of each agent will converge to the state of agent 1 and the consensus of the system will be reached fast.

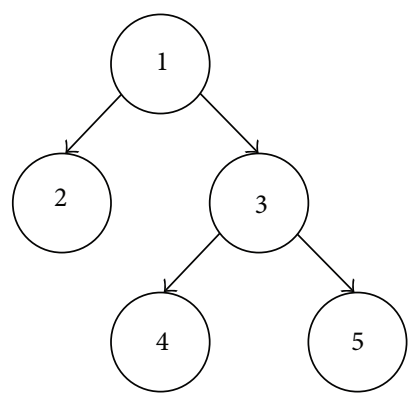

Figure 2: Interaction topology.

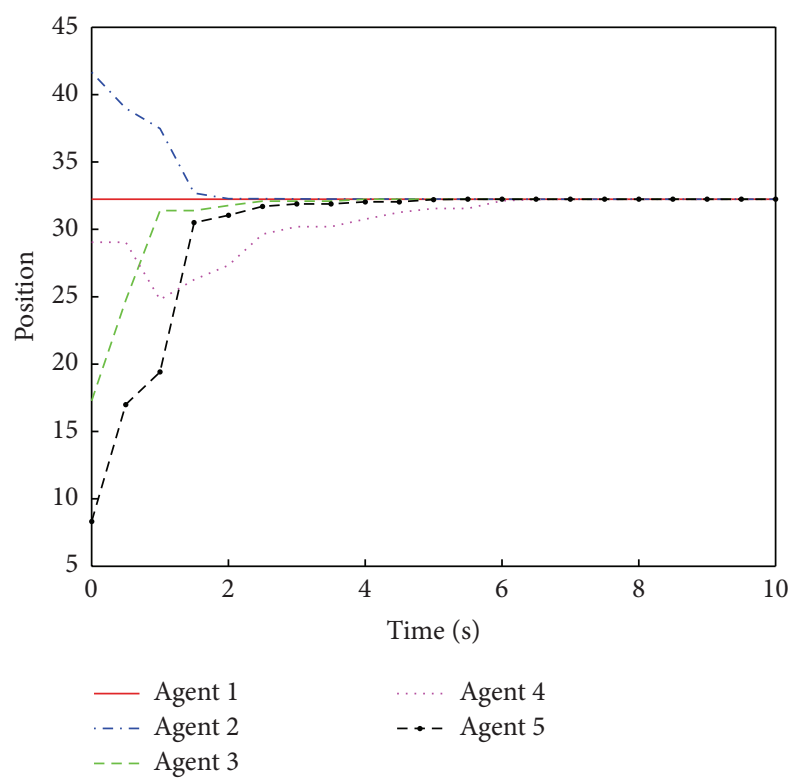

FIGURE 3: Consensus of single integrator system.

4.2. Second Order Agent. In this case, we need to test Schur stability of the error dynamics with Algorithm 1; it can be calculated that, with 582 subintervals, the conditions in Lemma 20 can be satisfied for every subinterval. Similar to Case 1, the initial values for position and velocity are randomly adopted within $[0,50]$ and $[0,10]$, respectively. The simulation results are shown in Figures 4 and 5 . It can be seen that, during some time intervals at the beginning, the difference of states may increase, especially for velocity of the agent, but the consensus will be reached with enough time. It is also worth noticing that the sampling and transmitting period $T$ adopted in this case cannot satisfy the condition in Theorem 17; however, the consensus can still be guaranteed which indicated that the condition from stochastic matrix theory is more conservative if Assumption 18 holds.

4.3. High-Order Agent. Considering the third order integrator agent, corresponding structure of matrices $A$ and $B$ can be obtained through Table 1. Then according to Lemmas 22 and 23, the controller gain $K$ can be calculated through solving the 5 LMIs with matlab. It can be found that $K=$ $\left[\begin{array}{lll}2.36 & 2.57 & 3.59\end{array}\right]$ is a proper solution and the simulations 


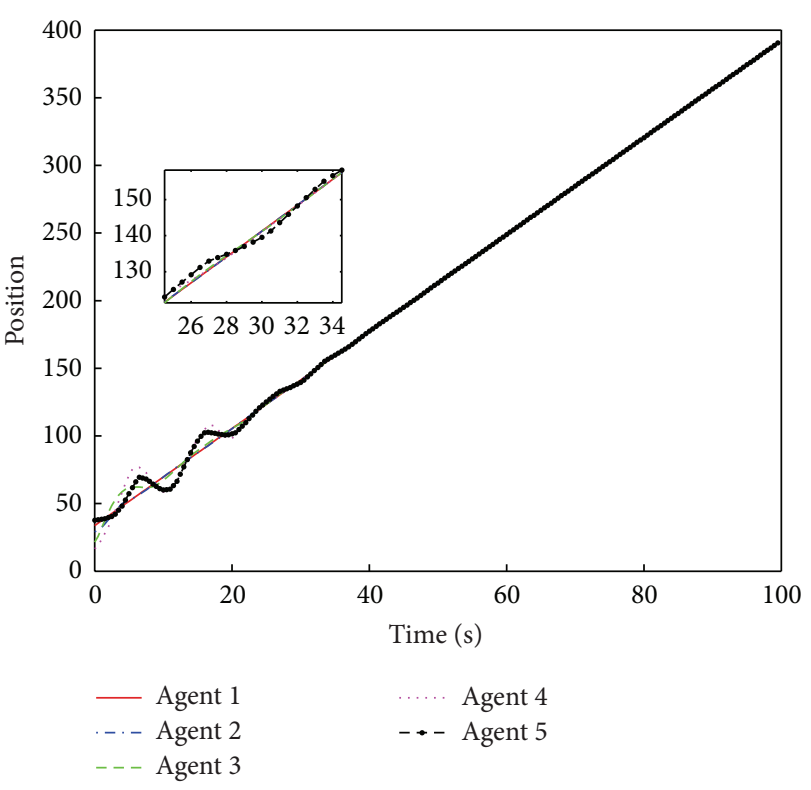

FIGURE 4: Consensus of position for second order system.

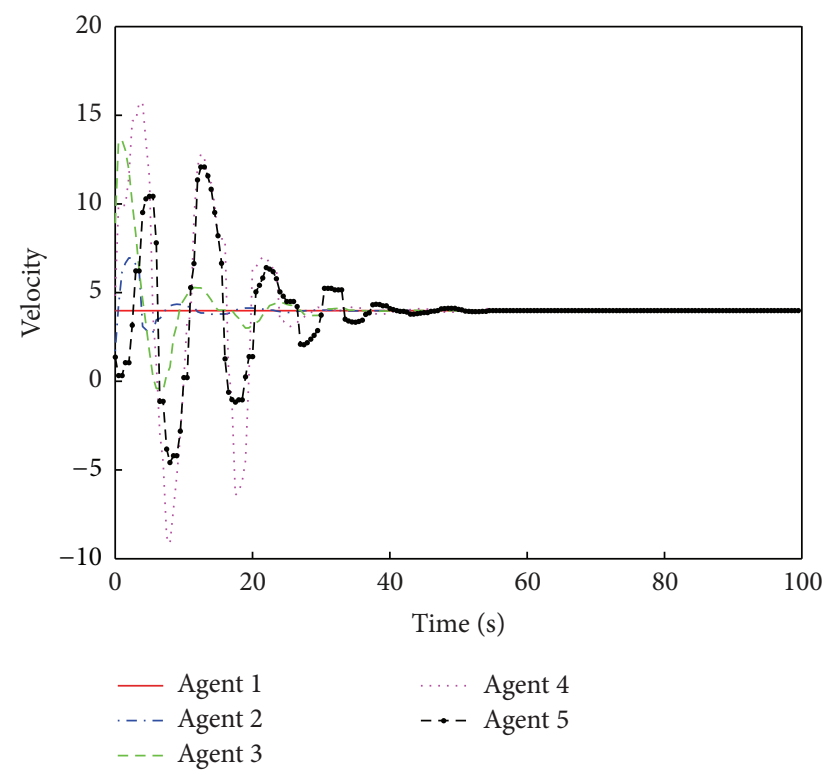

FIGURE 5: Consensus of velocity for second order system.

are carried out with results presented in Figures 6, 7, and 8. Even though it takes more time to reach the consensus for the multiagent system, with the proposed controller structure and controller gain, the consensusability is guaranteed. In addition, the initial states for position, velocity, and acceleration are randomly chosen from $[0,50],[0,10]$, and $[0,3]$.

\section{Conclusions}

In this paper, the consensus problem of multiagent system with packet losses and communication delays is discussed. A novel control protocol that depends on periodic sampling

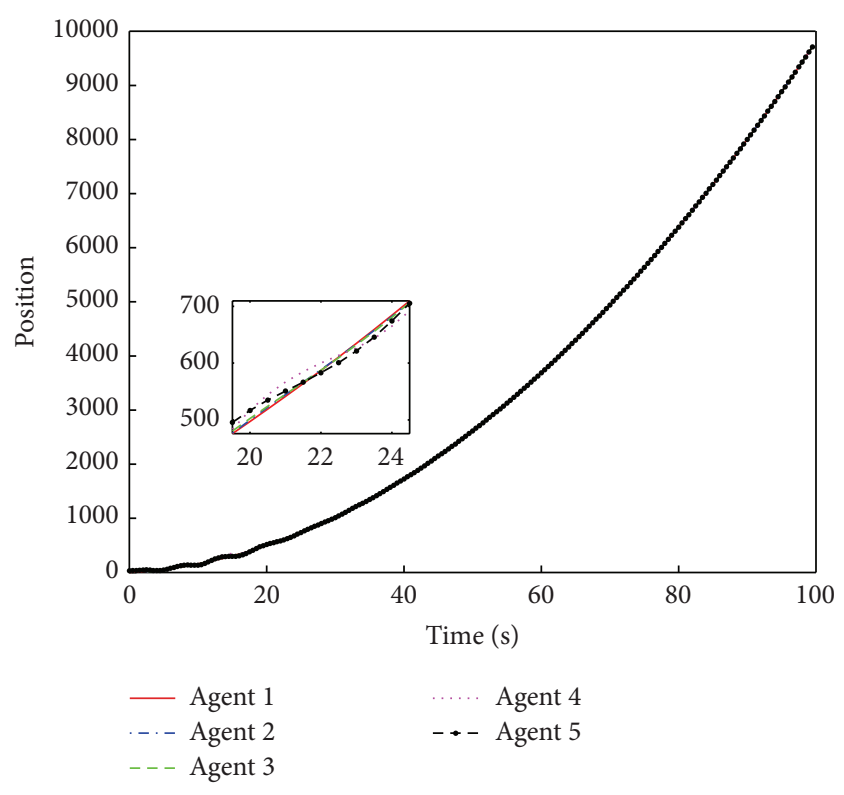

Figure 6: Consensus of position for high-order system.

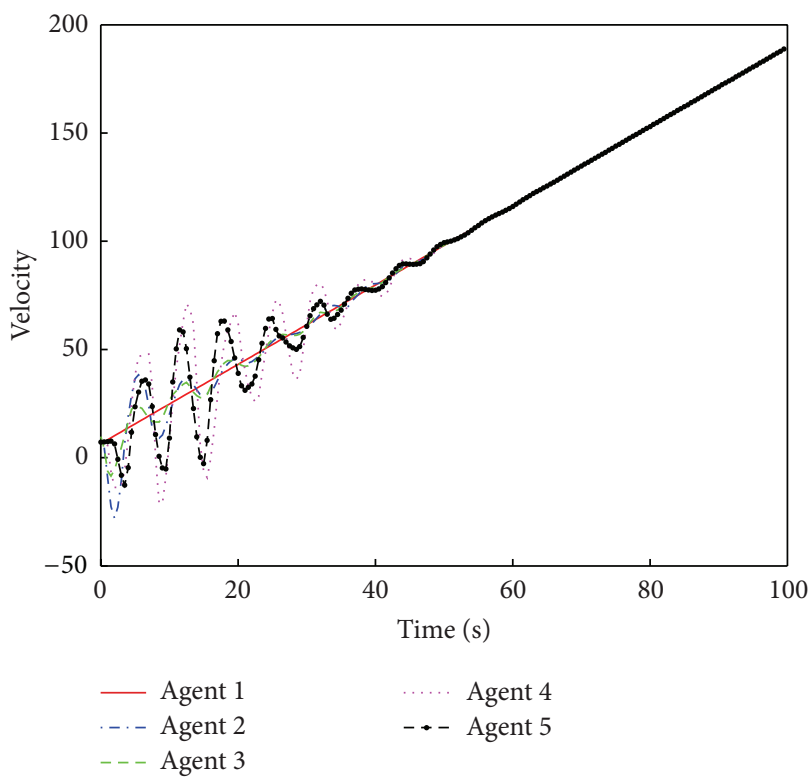

FIGURE 7: Consensus of velocity for high-order system.

and transmitting information is proposed to make it more convenient for implementation. Then, sufficient conditions for consensusability of multiagent systems consist of single integrator, second order, and high-order agents that are established, respectively, according to stochastic matrix theory and interval matrix theory. Finally, simulations are carried out to verify the theoretical results obtained. However, to reach the consensus for second and high-order systems with packet losses and communication delays, the directed communication topology is limited to a directed spanning tree. Even though this kind of structure has its own advantages in realworld applications, being convenient and economical, for 


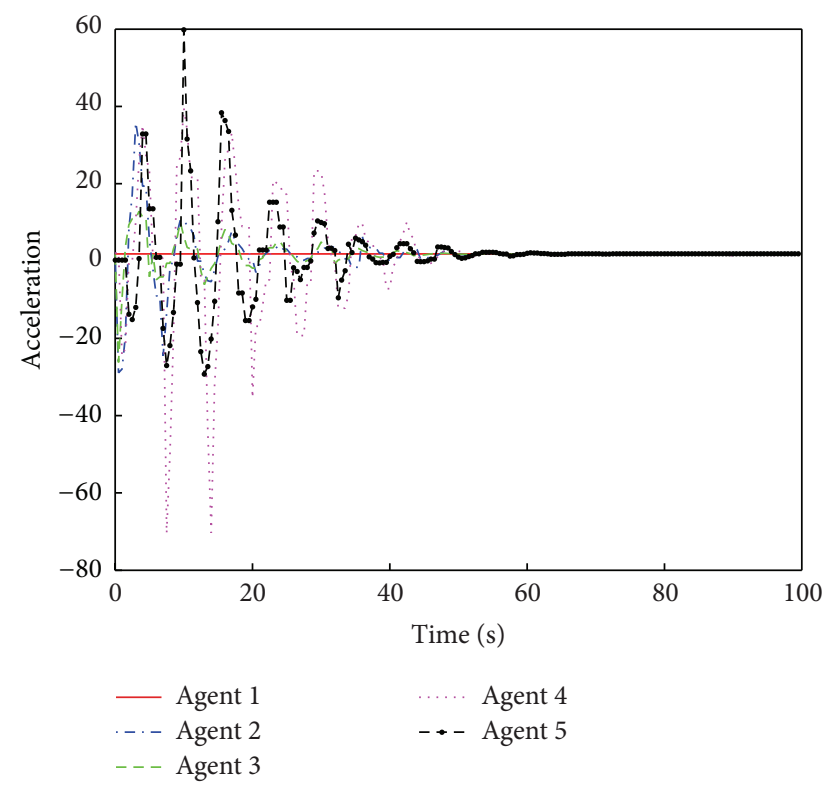

FIGURE 8: Consensus of acceleration for high-order system.

example, it also has some flaws in modeling the system with massive agents such as flock and fish school. Therefore, in the follow-up study of this research, the directed topology will not be limited to a directed spanning tree in order to make the whole system more distributive.

\section{Conflict of Interests}

The authors declare that they have no conflict of interests regarding the publication of this paper.

\section{Acknowledgments}

This work is supported by the National Natural Science Foundation of China (Grant nos. 5179038, 51109043, and 51309067) and New Century Excellent Talents in University of China (Grant no. NCET-10-0053).

\section{References}

[1] K. Nagatani, Y. Okada, N. Tokunaga et al., "Multirobot exploration for search and rescue missions: a report on map building in RoboCupRescue 2009," Journal of Field Robotics, vol. 28, no. 3, pp. 373-387, 2011.

[2] S. Zhang, J. Yu, A. Zhang, L. Yang, and Y. Shu, "Marine vehicle sensor network architecture and protocol designs for ocean observation," Sensors, vol. 12, no. 1, pp. 373-390, 2012.

[3] S. Iyengar and R. Brooks, Distributed Sensor Networks: Sensor Networking and Applications, CRC Press, 2012.

[4] R. Cui, S. S. Ge, B. Voon Ee How, and Y. Sang Choo, "Leaderfollower formation control of underactuated autonomous underwater vehicles," Ocean Engineering, vol. 37, no. 17-18, pp. 1491-1502, 2010.

[5] R. Olfati-Saber and R. M. Murray, "Consensus problems in networks of agents with switching topology and time-delays,"
IEEE Transactions on Automatic Control, vol. 49, no. 9, pp. 1520 1533, 2004

[6] Y. Zhang and Y.-P. Tian, "Consensus of data-sampled multiagent systems with random communication delay and packet loss," IEEE Transactions on Automatic Control, vol. 55, no. 4, pp. 939-943, 2010.

[7] W. Ni and D. Cheng, "Leader-following consensus of multiagent systems under fixed and switching topologies," Systems and Control Letters, vol. 59, no. 3-4, pp. 209-217, 2010.

[8] Y. Cao and W. Ren, "Sampled-data discrete-time coordination algorithms for double-integrator dynamics under dynamic directed interaction," International Journal of Control, vol. 83, no. 3, pp. 506-515, 2010.

[9] W. Ren and R. W. Beard, "Consensus seeking in multiagent systems under dynamically changing interaction topologies," IEEE Transactions on Automatic Control, vol. 50, no. 5, pp. 655661, 2005.

[10] Y. Gao and L. Wang, "Consensus of multiple double-integrator agents with intermittent measurement," International Journal of Robust and Nonlinear Control, vol. 20, no. 10, pp. 1140-1155, 2010.

[11] P. Lin, Z. Li, Y. Jia, and M. Sun, "High-order multi-agent consensus with dynamically changing topologies and timedelays," IET Control Theory and Applications, vol. 5, no. 8, pp. 976-981, 2011.

[12] P. Lin and Y. Jia, "Consensus of second-order discrete-time multi-agent systems with nonuniform time-delays and dynamically changing topologies," Automatica, vol. 45, no. 9, pp. 21542158, 2009.

[13] Y. Liu, H. Min, S. Wang, Z. Liu, and S. Liao, "Distributed adaptive consensus for multiple mechanical systems with switching topologies and time-varying delay," Systems \& Control Letters, vol. 64, pp. 119-126, 2014.

[14] Y. Gao, J. Ma, M. Zuo, T. Jiang, and J. Duc, "Consensus of discrete-time second-order agents with time-varying topology and time-varying delays," Journal of the Franklin Institute, vol. 349, no. 8, pp. 2598-2608, 2012.

[15] J. Zhu and L. Yuan, "Consensus of high-order multi-agent systems with switching topologies," Linear Algebra and Its Applications, vol. 443, pp. 105-119, 2014.

[16] J. Almeida, C. Silverstre, and A. M. Pascoal, "Continuous time consensus with discrete time communications," Systems \& Control Letters, vol. 61, no. 7, pp. 788-796, 2012.

[17] J. Qin, H. Gao, and W. X. Zheng, "Consensus strategy for a class of multi-agents with discrete second-order dynamics," International Journal of Robust and Nonlinear Control, vol. 22, no. 4, pp. 437-452, 2012.

[18] D. Goldin and J. Raisch, "Consensus for agents with double integrator dynamics in heterogeneous networks," Asian Journal of Control, vol. 15, no. 4, pp. 1-10, 2013.

[19] G. Parlangeli, "Collaborative diagnosis and compensation of misbehaving nodes in acyclic consensus networks: analysis and algorithms," International Journal of Innovative Computing Information and Control, vol. 9, no. 3, pp. 915-938, 2009.

[20] X. Su, L. Wu, Shi, and P. :, "Sensor networks with random link failures: distributed filtering for T-S fuzzy systems," IEEE Transactions on Industrial Informatics, vol. 9, no. 3, pp. 17391750, 2013.

[21] X. Su, X. Yang, P. Shi, and L. Wu, "Fuzzy control of nonlinear electromagnetic suspension systems," Mechatronics, 2013.

[22] X. Su, P. Shi, L. Wu, and Y.-D. Song, "A novel control design on discrete-time Takagi-Sugeno fuzzy systems with time-varying 
delays," IEEE Transactions on Fuzzy Systems, vol. 21, no. 4, pp. 655-671, 2013.

[23] J. Yu and L. Wang, "Group consensus in multi-agent systems with switching topologies and communication delays," Systems and Control Letters, vol. 59, no. 6, pp. 340-348, 2010.

[24] B. Iantovics and C. B. Zamfirescu, "ERMS: an evolutionary reorganizing multiagent system," International Journal of Innovative Computing Information and Control, vol. 9, no. 3, pp. 1171-1188, 2013.

[25] L. Wu, X. Su, and P. Shi, "Sliding mode control with bounded L2 gain performance of Markovian jump singular time-delay systems," Automatica, vol. 48, no. 8, pp. 1929-1933, 2012.

[26] R. A. Horn and C. R. Johnson, Matrix Analysis, Cambridge University Press, 2012.

[27] J. Wolfowitz, "Products of indecomposable, aperiodic, stochastic matrices," Proceedings of the American Mathematical Society, vol. 14, no. 5, pp. 733-736, 1963.

[28] F. Lewis, Cooperative Control of Multi-Agent Systems: Optimal and Adaptive Design Approaches, Springer, Berlin, Germany, 2013.

[29] A. Jadbabaie, J. Lin, and A. S. Morse, "Coordination of groups of mobile autonomous agents using nearest neighbor rules," IEEE Transactions on Automatic Control, vol. 48, no. 6, pp. 988-1001, 2003.

[30] K. Wang, A. N. Michel, and D. Liu, "Necessary and sufficient conditions for the Hurwitz and Schur stability of interval matrices," IEEE Transactions on Automatic Control, vol. 39, no. 6, pp. 1251-1255, 1994.

[31] X. Liao, Q. Luo, Z. Mei, and W. Hu, "Notes on necessary and sufficient conditions of stability, observability and controllability for interval matrices," Acta Automatica Sinica, vol. 24, no. 6, pp. 829-833, 1998.

[32] D.-Q. Zhang, Q.-L. Zhang, and Y.-P. Chen, "Controllability and quadratic stability quadratic stabilization of discrete-time interval systems-an LMI approach," IMA Journal of Mathematical Control and Information, vol. 23, no. 4, pp. 413-431, 2006. 


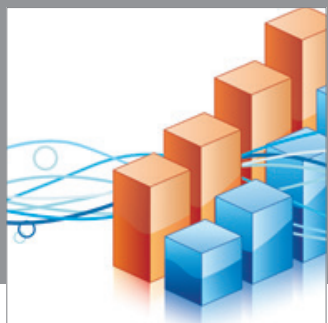

Advances in

Operations Research

mansans

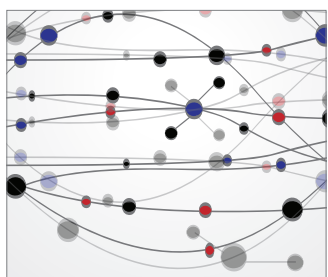

The Scientific World Journal
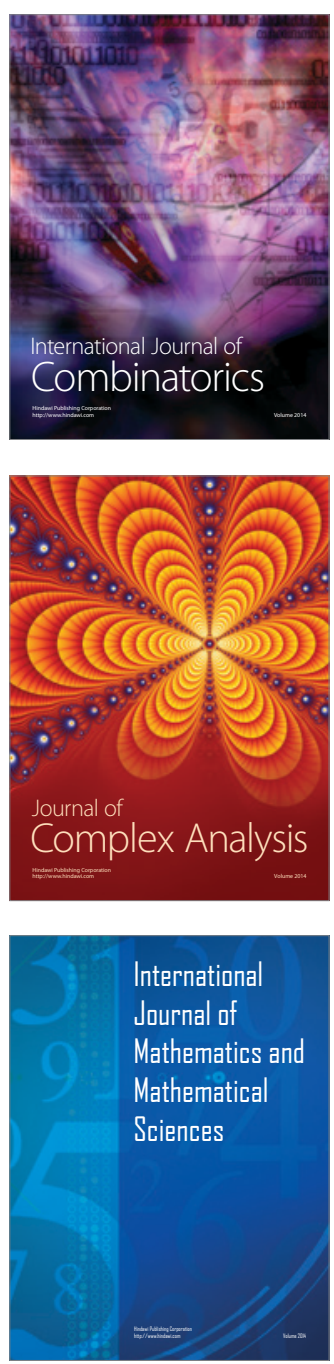
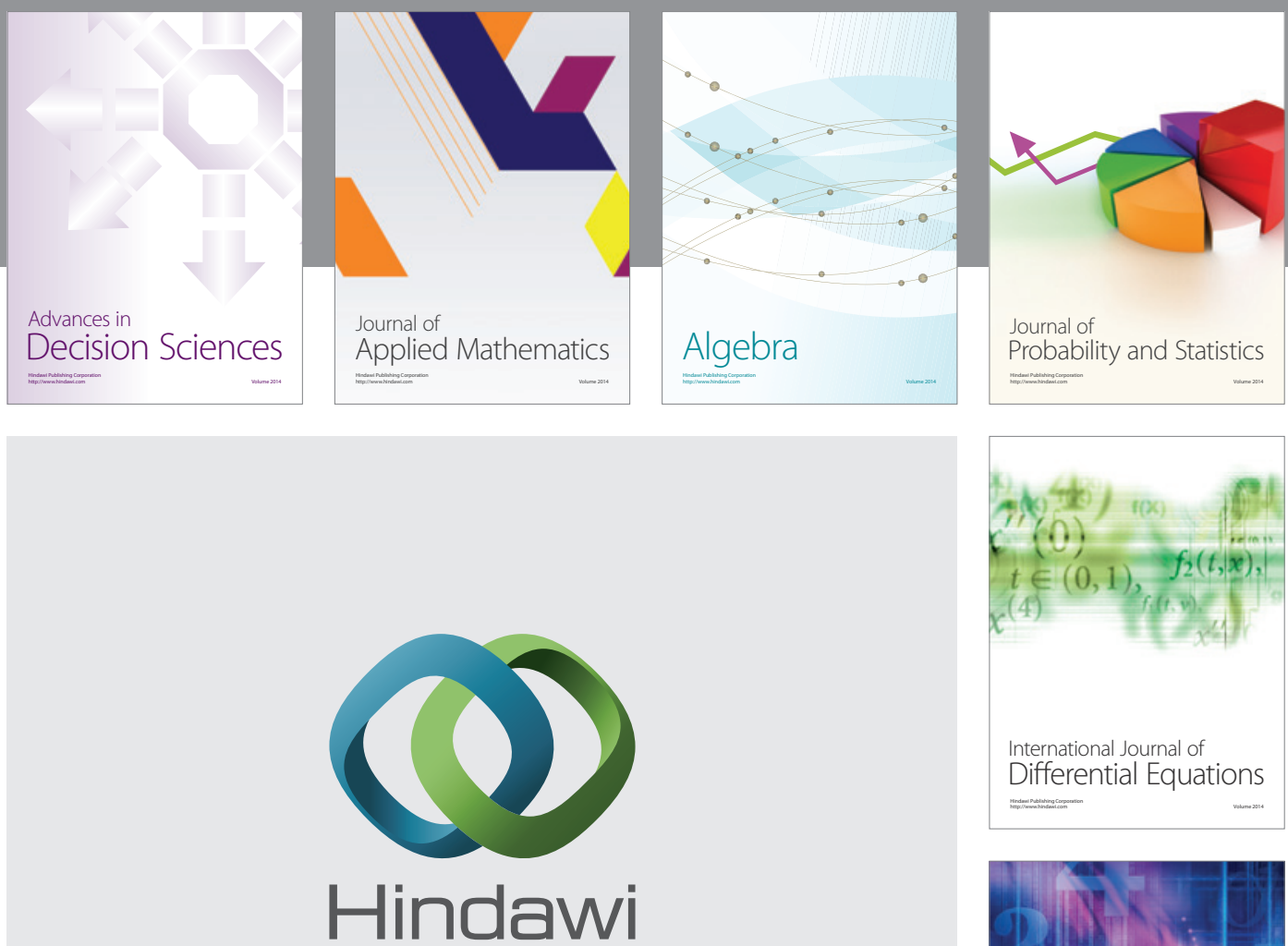

Submit your manuscripts at http://www.hindawi.com
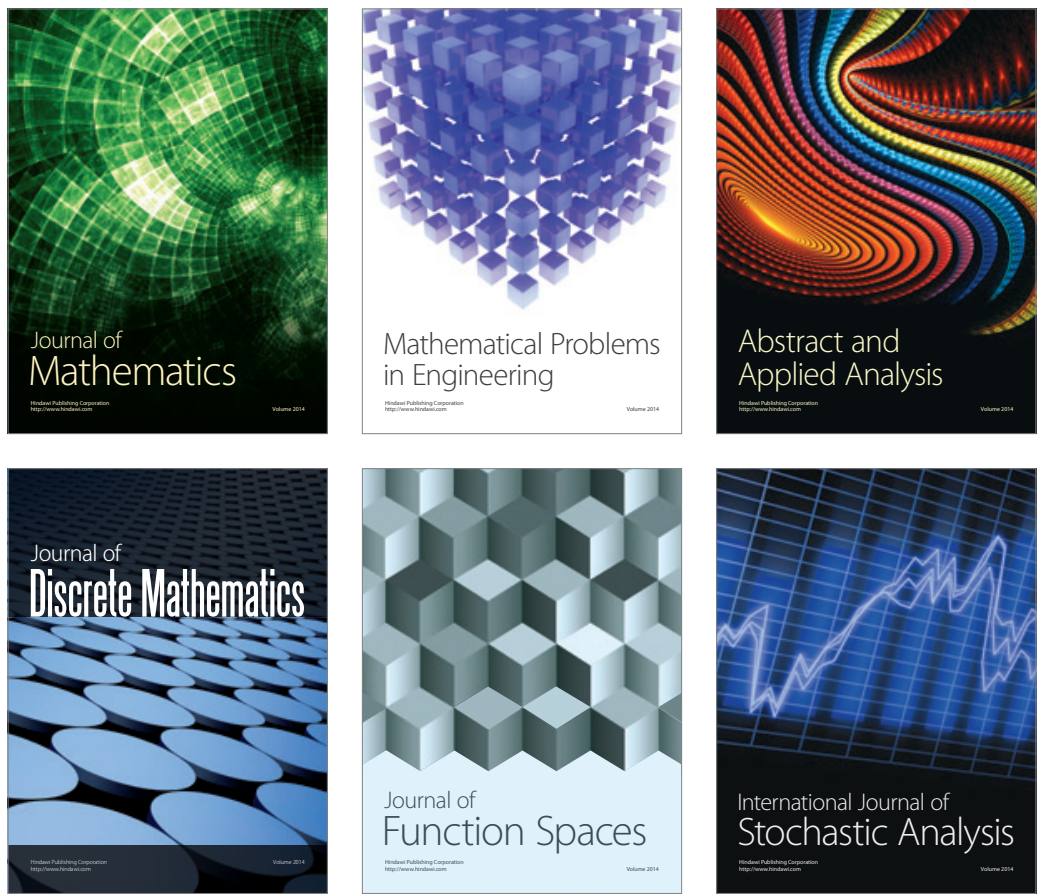

Journal of

Function Spaces

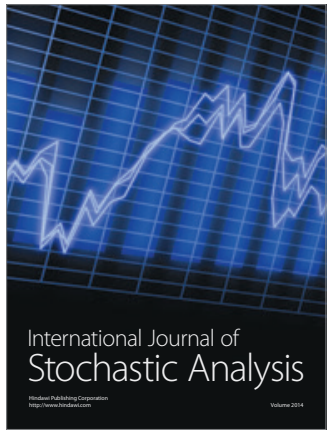

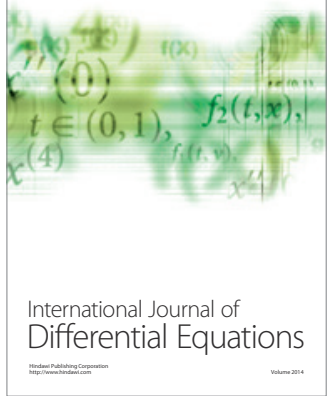
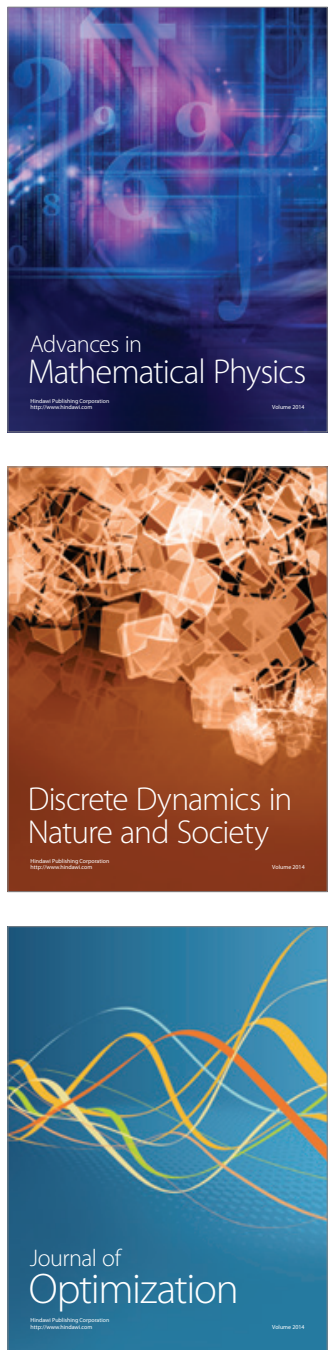